\title{
IMPROVED RATIONAL TWO-PORT MODEL OF UNIFORM R-C-NR STRUCTURE
}

\author{
UMESH KUMAR and SOMESH JHA \\ Department of Electrical Engineering, Indian Institute of Technology, Hauz Khas, New Delhi - 110016, India
}

(Received November 2, 1984; in final form November 14, 1984)

The indefinite admittance matrix of the uniform R-C-NR structure (1) is written in a modified manner. The new expressions for the admittance parameters are expanded utilizing the technique postulated earlier by Dutta Roy and Kumar (2). A rational two-port lattice equivalent of the uniform R-C-NR structure is constructed. This proposed model is much more accurate over a much wider frequency range and is very much improved because it stimulates the characteristics more precisely and extensively.

\section{INTRODUCTION}

Distributed parameter circuits find wide applications because they offer the advantages of miniaturized size, relative ease of controlling the circuit parameters, low cost and high reliability compared with lumped circuits. Various multilayered structures have been studied by many workers. Different types of electrical performance characteristics may be obtained from such a structure by imposing various sets of constraints on its terminals and generating Subnetworks(3). Castro and Happ (1) described the use of R-C-NR structure (Fig. 1) for realizing various transformation functions e.g. notch filter, bandpass, band reject and high pass filters.

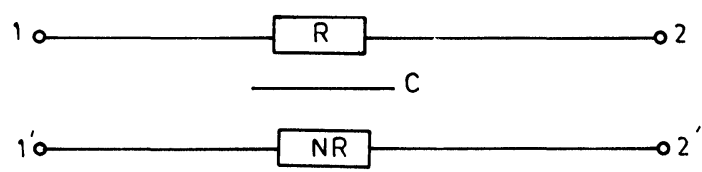

FIGURE 1 Symbolic representation of a uniform R-C-NR Structure.

\section{ASSUMPTIONS MADE DURING THE DEVELOPMENT}

The authors, while developing the theoretical model, have only assumed the transmission line model of the R-C-NR structure. Kamal et al (4) derived the indefinite admittance matrix in agreement with the results obtained by Castro and Happ by taking a one dimensional transmission line as a mathematical representation (5). This model is valid because of two reasons. First we have assumed (and which is mostly so) that breadth and thickness of the structure are very much smaller than its length. Secondly, based on this model many workers have made successful devices. Some of the workers who have made successful devices are Kamal et al(4), V.P. Gupta et al (10), V.P. Gupta et al (9) and K. Pal (11). The other concepts used are well proven mathematical facts. 


\section{DEVELOPMENT OF THE MODEL}

The use of the general transmission line model poses a formidable problem and involves considerable mathematical intricacy and complexity. This arises from the fact that an infinite number of sections of elements are involved. Even if the number of sections is limited, an impractically large number is required for achieving tolerable accuracy. Simulation of the model necessitates prohibitive computational labour.

Kumar and Pal (6) have attempted modelling the uniform R-C-NR structure by developing a second order two-port model which approximates the actual characteristics very closely over a wide frequency range $\left(0<\theta<20, \theta=[(1+N) \omega R C]^{1 / 2}\right)$. The model has been constructed utilizing the continued fraction expansion and suitable truncation of the short circuit admittance parameters (2).

From mathematical considerations, it has been found that the admittance parameters can be written purely as functions of $\operatorname{coth}(\theta)$, which has got a more accurate continued fraction expansion than $\operatorname{Cosech}(\theta)(7)$. The modified expression for the parameters lead to an improved model of the structure.

It is known (8) that the equivalent circuit of a bilateral four terminal network can be constructed in the form of a rectangular lattice with its arms containing admittances as shown in Fig. 2.

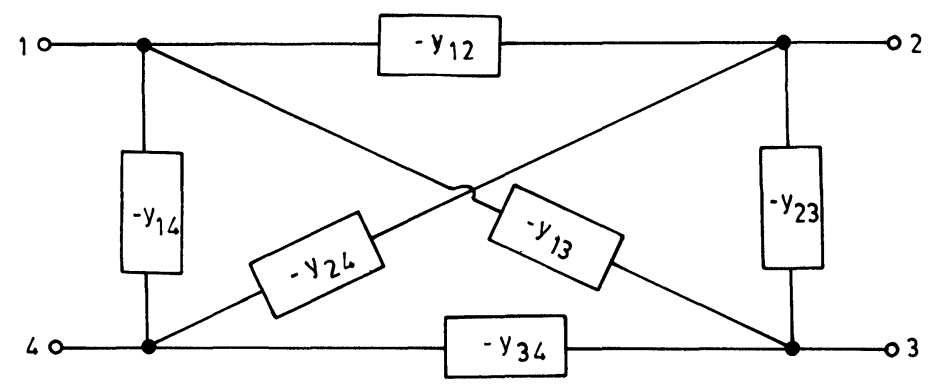

FIGURE 2 General lattice equivalent of a bilateral four-terminal Network.

The admittance parameters necessary and sufficient for development of the model of uniform R-C-NR structure from its indefinite admittance matrix are:

$$
\begin{aligned}
& \mathrm{y}_{12}=-\frac{1}{(1+\mathrm{N}) \mathrm{R}}(\theta \operatorname{cosech}(\theta)+\mathrm{N}) \\
& \mathrm{y}_{13}=\mathrm{y}_{24}=\frac{1}{(1+\mathrm{N}) \mathrm{R}}(\theta \operatorname{cosech}(\theta)-1) \\
& \mathrm{y}_{23}=\mathrm{y}_{14}=\frac{1}{(1+\mathrm{N}) \mathrm{R}}(1-\theta \operatorname{coth}(\theta)) \\
& \mathrm{y}_{34}=\frac{-1}{(1+\mathrm{N}) \mathrm{R}} \quad \theta \operatorname{cosech}(\theta)+\frac{1}{\mathrm{~N}}
\end{aligned}
$$

where $\theta=[1+\mathrm{N}) \mathrm{SRC}]^{1 / 2}$ 
and $\mathrm{s}$ is the complex frequency variable.

The rest of the admittances contained in the indefinite admittance matrix are not needed in this representation, being equal to one or the other parameters mentioned above. In terms of $\operatorname{coth}(\theta)$ only the modified expressions for these parameters are obtained by substituting (7) for.

$\theta \operatorname{cosech}(\theta)=\left[\frac{\theta}{2} \operatorname{coth}(\theta / 2)-\frac{\theta^{2} / 4}{(\theta / 2) \operatorname{coth}(\theta / 2)}\right]$

Now these parameters involve hyperbolic functions of a complex irrational argument. Synthesis of a single section, lumped, two-port model with simple elements is impossible from these parameters in such a form. As such, using the method expounded in (2), the $\operatorname{coth}(\theta)$ and $\operatorname{coth}(\theta / 2)$ terms are expanded in a continued fraction expansion and truncated at appropriate terms as given below:

$\theta \operatorname{coth}(\theta)=\theta\left(1 / \theta+1\left(\frac{3}{\theta}+1 \quad\left(\frac{5}{\theta}+1\left(\frac{7}{\theta}\right)\right)\right)\right)$

$\left(\frac{\theta}{2}\right) \operatorname{coth}\left(\frac{\theta}{2}\right)=\frac{\theta}{2}\left(\frac{2}{\theta}+1\left(\frac{6}{\theta}+1\left(\frac{10}{\theta}+1\left(\frac{14}{\theta}\right)\right)\right)\right)$

Substituting for $\theta$ and replacing the hyperbolic function by these, rational approximations to the parameters are obtained as:

$$
\begin{aligned}
&-\mathrm{y}_{12}=\frac{1}{\mathrm{R}}+ {\left[1\left(\frac{12}{\mathrm{SC}}+1\left(\frac{5}{(1+\mathrm{N}) \mathrm{R}}+1\left(\frac{28}{\mathrm{SC}}\right)\right)\right)\right] } \\
&-\left[\frac{1}{\frac{4(1+\mathrm{N})}{\mathrm{SC}}+1\left(\frac{3}{(1+\mathrm{N}) \mathrm{R}}+1\left(\frac{20}{\mathrm{SC}}+1\left(\frac{7}{(1+\mathrm{N}) \mathrm{R}}\right)\right)\right)}\right] \\
&-\mathrm{y}_{13}=-\mathrm{y}_{12}-\frac{1}{\mathrm{R}} \\
&-\mathrm{y}_{34}=-\mathrm{y}_{12}-\frac{1}{\mathrm{R}}+\frac{1}{\mathrm{NR}}
\end{aligned}
$$

and

$$
-\mathrm{y}_{14}=\left[1\left(\frac{3}{\mathrm{SC}}+1\left(\frac{5}{(1+\mathrm{N}) \mathrm{R}}+1\left(\frac{7}{\mathrm{SC}}\right)\right)\right)\right]
$$


Straightforward Cauer synthesis from the admittance expressions above results in the new and accurate lattice equivalent of the R-C-NR structure as in Fig. 3.

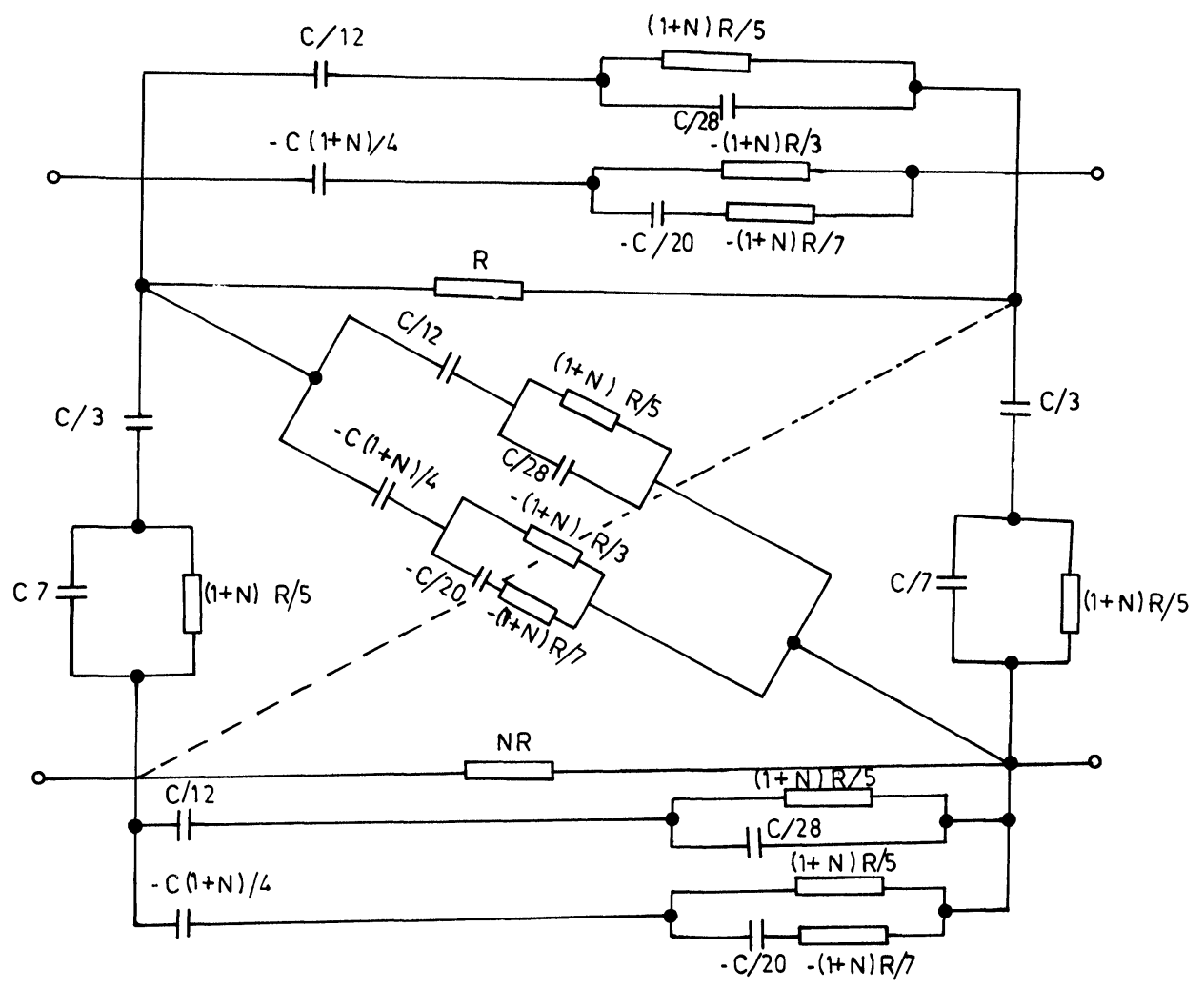

FIGURE 3 The proposed model.

\section{CONCLUSION}

The model is deemed to be extremely versatile, powerful and useful e.g. it may be applied to predict precisely the behaviour of various filter configurations, band pass amplifier, phase shift oscillator etc. which can be formed using a R-C-NR structure. The use of R-C-NR structure eliminates the need to employ lumped elements together with two layer distributed structures to realize various circuit functions.

This method can easily lead to computerization. Using this model a comprehensive software package for various devices made by R-C-NR structure can easily be designed. As all the elements in the circuit are lumped the effect of random errors during fabrication can easily be taken into account. The variation of various parameters with frequency and temperature can easily be studied. So this model can lead to effective sensitivity analysis of the devices made by R-C-NR structure.

The proposed model (Fig. 3 ) is much more accurate than the model proposed earlier (6) because it utilizes only the $\operatorname{coth}(\theta)$ expansion. Further to its accuracy range is considerably increased to o $<\theta<80$ since expansions are now made in terms of $\theta^{2} / 4$ rather than $\theta^{2}$. It may be noted that, for $\mathrm{N}=0$, the model reduces to that given earlier (2) as it should. 


\section{REFERENCES}

1. P.S. Castro and W.W. Happ, "Distributed Parameter circuit and microsystem electronics", Proceedings of the national electronics conference (Chicago, USA), 1960, Vol. 16, pp. 448-460.

2. S.C. Dutta Roy and U. Kumar, "A rational two-port model of the uniformly distributed RC structure, IEEE transaction on circuits and systems, Vol. CAS-23, pp. 56-58, January 1976.

3. P.S. Castro and W.W. Happ, "Subnetworks", IRE International convention Record (New York) USA. Vol. 10, part 2, pp. 3-7, March 1962.

4. A.K. Kamal, K.U. Ahmed and R.P. Agarwal, "Performance characteristics of uniform thin film resistivecapacities resistive structures", Microelectronics J., Vol. 6, no. 1, pp. 6-10, 1974.

5. M.S. Ghausi and J.J. Kelly, Introduction to distributed parameters network with application to integrated circuits, Holt Rinehart and Winston, New York, USA, 1968.

6. U. Kumar and K. Pal, "Accurate single section model of uniform R-C-NR structure", Electronics letters, Vol. 14, no. 12, pp. 384-385, 8th June 1978.

7. U. Kumar and K. Pal, "Modified rational two-port model of the uniformly distributed RC structure, Archiv für Electronik and U bertragungstechnik, Band 34, Heft 11, pp. 471-472, 1980.

8. E.V. Zelyakh, "An equivalent circuit for a doubly symmetrical $5 \times 1$ terminal network", Radio Engg. and Electronics Physics, Vol. 19, no. 3, pp. 73-78, March 1974.

9. V.P. Gupta, K. Pal and A.K. Gupta, "An Evaporated Multilayer thin film RC Notch Filter," Thin solid films, Vol. 87, pp. 293-296, 1982.

10. V.P. Gupta, K. Pal and P.N.L. Gupta, "Evaporated thin films RC filters", Microelectron. and Reliab., Vol. 21, No. 4, pp. 529-532, 1981.

11. K. Pal, J. IETE, Vol. 24, pp. 374, September 1978. 

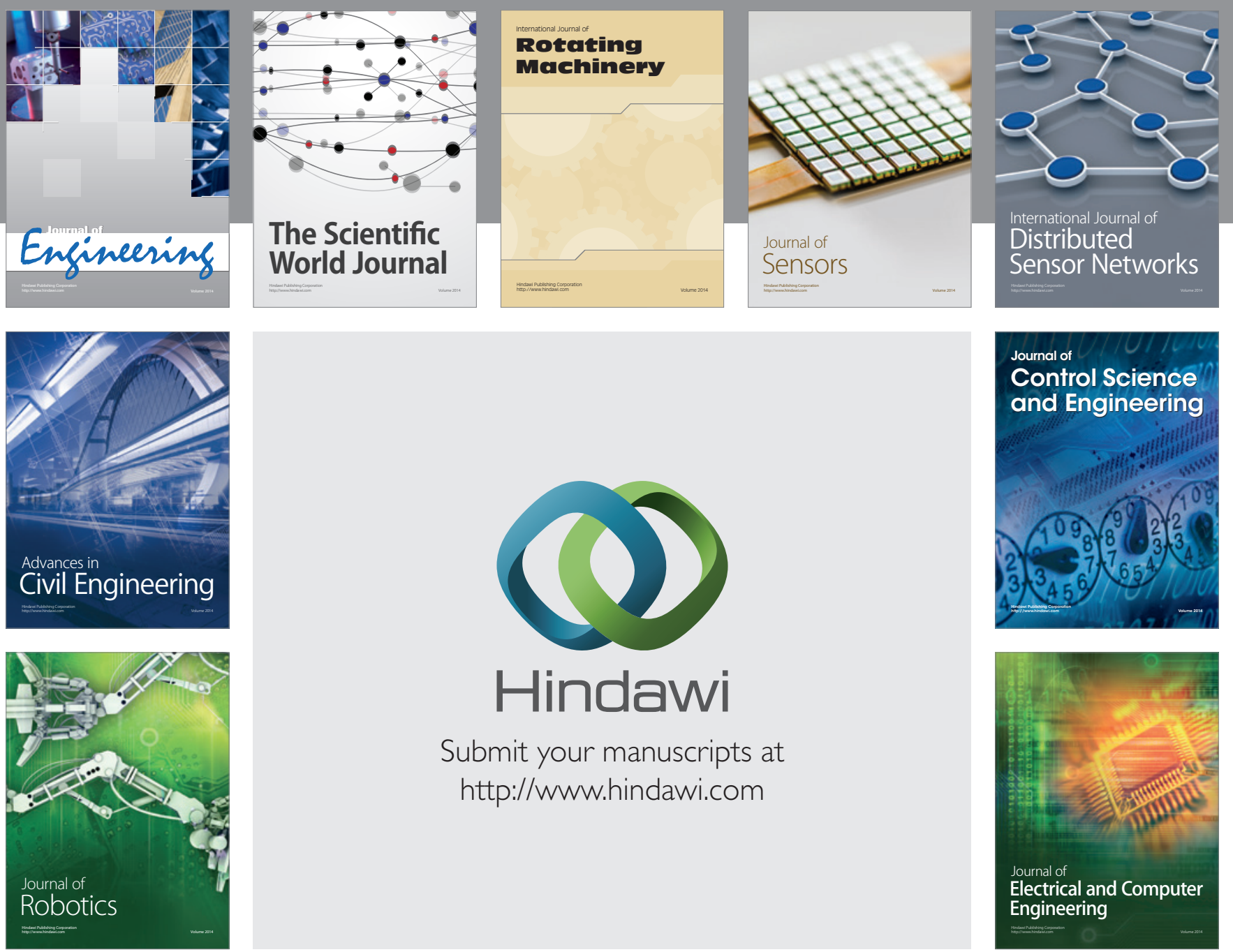

Submit your manuscripts at

http://www.hindawi.com
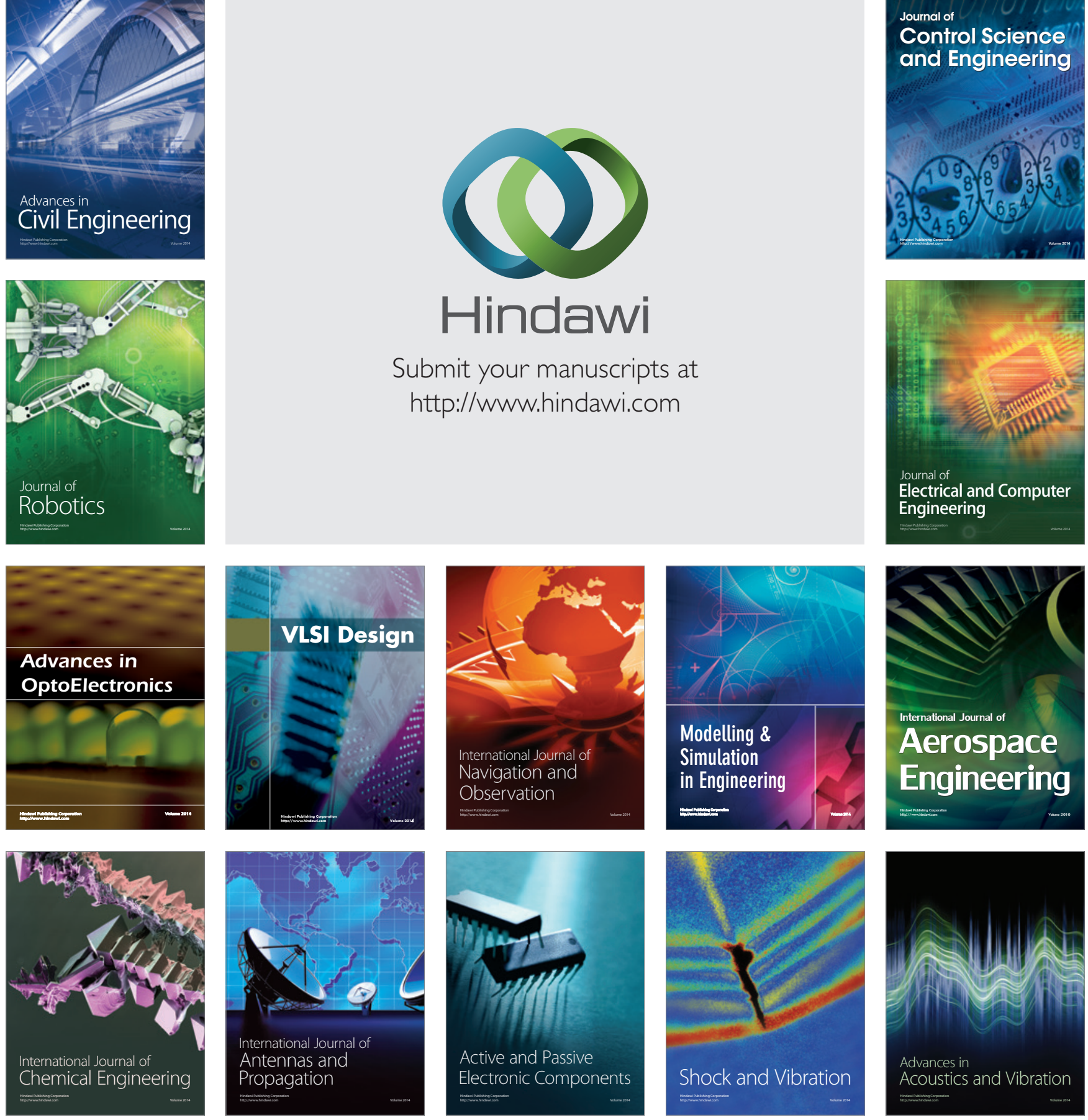